\begin{tabular}{|c|c|c|}
\hline $\begin{array}{c}\text { PRAMANA } \\
-\begin{array}{c}\text { journal of } \\
\text { physics }\end{array}\end{array}$ & (C) Indian Academy of Sciences & $\begin{array}{l}\text { Vol. } 53 \text {, No. } 3 \\
\text { September } 1999 \\
\text { pp. } 541-544\end{array}$ \\
\hline
\end{tabular}

\title{
Inclusive and exclusive measurements in the projectile breakup of ${ }^{7} \mathbf{L i}$
}

\author{
T MADHUSOODHANAN ${ }^{1}$, SAMIT MANDAL $^{2}$, M P SATHYAVATHIAMMA ${ }^{1}$, \\ RAMANI $^{1}$, N G PUTTASWAMY ${ }^{1}$, T S MUDHOLE ${ }^{3}$, A MANDAL $^{2}$, D K AVASTHI ${ }^{2}$, \\ R SHYAM $^{4}$ and S K DATTA ${ }^{2}$ \\ ${ }^{1}$ Department of Physics, Bangalore University, Bangalore 560 056, India \\ ${ }^{2}$ Nuclear Science Centre, P.B. No 10502, New Delhi 110 067, India \\ ${ }^{3}$ Department of Physics, Karnatak University, Dharwad 580 003, India \\ ${ }^{4}$ Saha Institute of Nuclear Physics, Calcutta 700 064, India
}

\begin{abstract}
The inclusive and exclusive measurements were carried out for ${ }^{7} \mathrm{Li}$ projectile breakup on ${ }^{27} \mathrm{Al}$ target at $48 \mathrm{MeV}$. In the inclusive data we have observed a broad peak around the beam velocity for alphas and tritons. The exclusive data for alpha-triton coincidences show good agreement with the post-form DWBA theory of breakup reactions.
\end{abstract}

Keywords. Direct reactions; projectile breakup; post-form DWBA.

PACS Nos 25.10+s; 24.50+g; 25.70+z

\section{Introduction}

There has been considerable interest in the study of the emission of charged particles in a reaction between two complex nuclei. It is characterized by large cross-sections and unusual shapes of the energy spectra. In addition to the compound nucleus formation and emission of charged particles from the equilibrated system, the direct breakup of the projectile in the field of the target nucleus is found to be a dominant reaction channel [1-3]. A broad peak centered around the beam velocity is characteristic of the projectile breakup. The inclusive spectra are basically interpreted to originate from fragments in a spectator role, reflecting the momentum distribution of the clusters in the projectile before collision. Various simple models describe the shapes of the breakup spectra fairly well [4]. However these models account for only the elastic breakup mode and neglect the inelastic mode. Experimentally it is found that the inelastic channel contributes predominantly to the inclusive cross-section. A realistic approach is the DWBA breakup theory developed by Baur and coworkers. The theory is based on a one-step breakup mechanism and enables calculations of contributions from both the elastic and inelastic breakup modes [5].

A kinematically complete study of the ${ }^{7} \mathrm{Li}$ breakup is of considerable importance as it may lead to a better understanding of the breakup of the neutron rich ${ }^{11} \mathrm{Li}$ isotope and 
other isotopes which have a 'halo' structure [6]. Our motivation in this study is mainly to investigate the nuclear and Coulomb part of the breakup cross section.

\section{Experimental details}

A beam of $48 \mathrm{MeV}{ }^{7} \mathrm{Li}$ obtained from the Nuclear Science Centre, New Delhi was used in the experiment. A self supporting ${ }^{27} \mathrm{Al}$ foil of thickness $190 \mu \mathrm{g} / \mathrm{cm}^{2}$ was used as target. The particle identification was achieved by using $\Delta E-E$ detector telescope. The experiment was carried out in the $1.5 \mathrm{~m}$ diameter general purpose scattering chamber (GPSC). Two telescopes were kept on each arm of the GPSC and one telescope was moved to different angles with the other fixed at $10^{\circ}$. The solid angle subtended by the solid state detector was $0.3 \mathrm{msr}$. The beam current on the target was around $10 \mathrm{pnA}$. A monitor detector was kept at $10^{\circ}$ to view the beam. The data were also acquired using both solid state detectors (SSD) and a combination of a gas ionization chamber and a position sensitive strip detector (PSSD). The SSDs used were $100 \mu \mathrm{m} \mathrm{Si}$ for $\Delta E$ detector and $5 \mathrm{~mm} \mathrm{Si(Li)} \mathrm{for} \mathrm{the} E$ detector. The $1 \mathrm{~mm}$ thick silicon PSSD with an active area of $50 \mathrm{~mm} \times 50 \mathrm{~mm}$ was used as $E$ detector. An ionization chamber with an active length of $10 \mathrm{~cm}$, and operated at a field of $1 \mathrm{~V} / T o r r / \mathrm{cm}$, was used as a $\Delta E$ detector for alpha particles. The coincidences between alpha particles and tritons were also recorded.

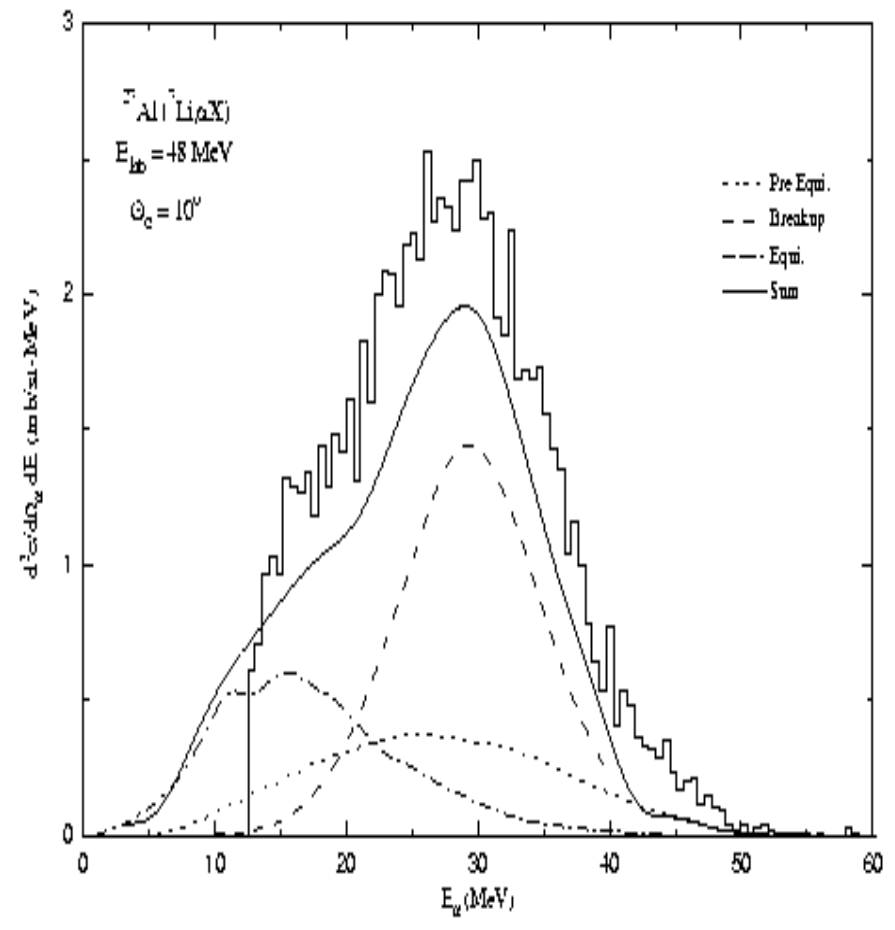

Figure 1. Experimental alpha energy (shown as histogram) spectrum obtained at $10^{\circ}$ in the reaction ${ }^{7} \mathrm{Li}$ on ${ }^{27} \mathrm{Al}$ at $48 \mathrm{MeV}$. 


\section{Inclusive measurements and results}

The differential energy spectra of the outgoing alphas and tritons were measured over a range of $8^{\circ}$ to $38^{\circ}$ in steps of $2^{\circ}$. A typical alpha spectrum at $10^{\circ}$ is shown in figure 1 . The broad bump corresponding to the beam velocity can be seen at the alpha energy of 27.4 $\mathrm{MeV}$.

The breakup contribution was calculated in the framework of post-form DWBA theory. The inclusive breakup cross section for the reaction $a+A \rightarrow b+x+B$, with $c=B+x$, can be expressed as

$$
\mathrm{d} \sigma^{\mathrm{incl}}=\frac{(2 \pi)^{4}}{\hbar v_{a}} \sum_{c}\left|T_{a, b c}^{\mathrm{DWA}}\right|^{2} \delta\left(E-E_{b}-E_{B c}\right) \mathrm{d}^{3} q_{b},
$$

where $v_{a}$ denotes the projectile velocity [5]. In this calculation for the breakup of ${ }^{7} \mathrm{Li}$, a value of $-100 \mathrm{MeV} . \mathrm{fm}^{3 / 2}$ has been used for $D_{0}$. The prediction of the direct breakup theory is shown in figure 1 as dashed line. In order to take into account the high energy tail, the pre-equilibrium contribution has been estimated in the frame work of the coalescence model using a coalescence radius of $248 \mathrm{MeV} / \mathrm{fm}$ for alpha particles [7]; this is shown as dotted line. The low energy part of the spectrum is accounted for by the equilibrium emission of the particles. The energy distribution of those particles is calculated using the PACE code and the result is shown as dash-dotted curve. The sum of all these three contributions shown as solid line gives a reasonable fit to the experimental spectrum.

\section{Exclusive measurements and results}

For coincidence measurements one telescope was fixed at $10^{\circ}$ and the other moved to different angles. The $E_{\alpha}+E_{t}$ spectrum showed a quasi-elastic peak around $45 \mathrm{MeV}$. This peak is used as a gate for extracting the exclusive spectra. It was noticed that there were no prominent peaks arising from the resonant breakup of ${ }^{7} \mathrm{Li}$ especially via the $4.63 \mathrm{MeV}$ state. The energy integrated triple differential cross sections for alpha particles extracted at each angle are shown in figure 2 .

These cross sections were compared with the direct fragmentation model (DFM) of breakup reactions formulated within the post-form DWBA [5]. In this model the incoming projectile is assumed to breakup instantaneously in the Coulomb and nuclear fields of the target nucleus. The triple differential cross section is given by

$$
\frac{\mathrm{d}^{3} \sigma}{\mathrm{d} \Omega_{b} \mathrm{~d} \Omega_{x} \mathrm{~d} E_{b}}=\rho\left|T_{f i}^{\mathrm{DWBA}}\right|^{2},
$$

where $\rho$ is the three-body density of states [5]. The optical potentials needed for the calculations are taken from the literature. The value of $D_{0}$ used in the evaluation of the matrix element is given in $\S 3$. In figure 2 , the dashed (dotted) line represents the results of the calculation preformed with pure nuclear (pure Coulomb) interactions while the solid line is the full DWBA calculation. The theory is able to quantitatively describe the features of the data in the region where the angular seperation between the fragments is large. A similar agreement has been found for the case of energy integrated triton cross-sections. 


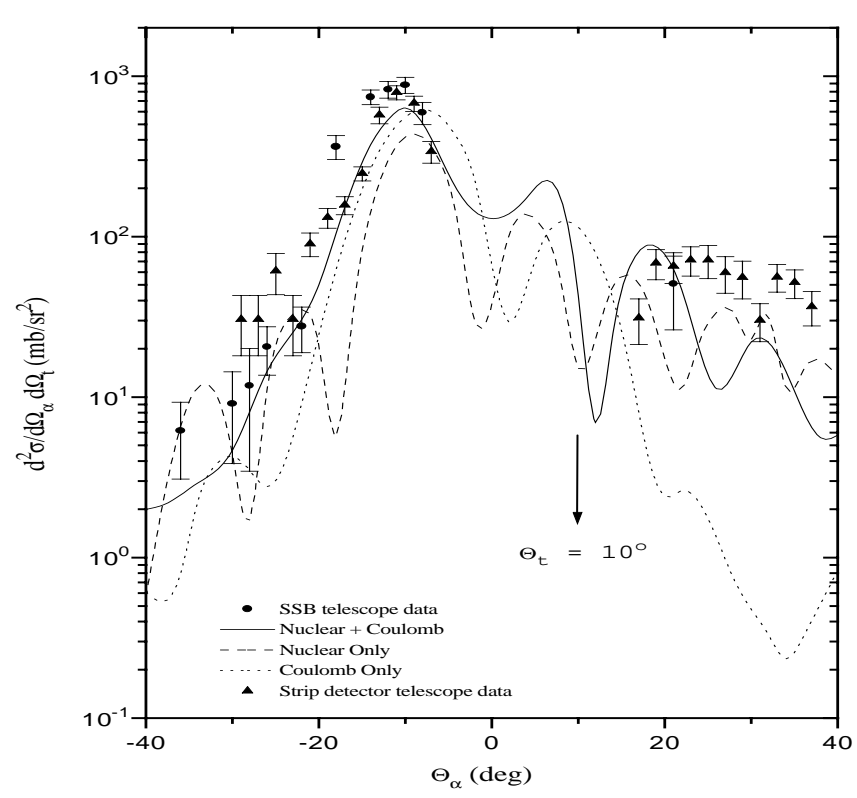

Figure 2. Energy integrated triple differential cross sections for alpha particles in the ${ }^{7} \mathrm{Li}$ breakup on ${ }^{27} \mathrm{Al}$ at $48 \mathrm{MeV}$. The triton detector was fixed at $10^{\circ}$.

\section{Acknowledgement}

The authors wish to thank the Pelletron staff of NSC for smooth running of the machine. The project has been partially supported by a research grant from the University Grants Commission.

\section{References}

[1] J Pampus, J Bisplinghoff, J Ernst, T Mayer-Kuckuk, J Rama Rao, G Baur, F Rosel and D Trautmann, Nucl. Phys. A311, 141 (1978)

[2] N Matsuoka, A Shimizu, K Hosono, T Saito, M Kondo, H Sakaguchi, A Goto and F Oklani, Nucl. Phys. A337, 269 (1980)

[3] B Neumann, H Rebel, J Buschmann, H J Gils, H Klewe-Nebenius and S Zagromski, Nucl. Phys. A269, 113 (1980)

[4] U Bechstedt, H Machner, G Baur, R Shyam, C Alderliesten, O Bousshid, A Djaloeis and P Jahn, Nucl. Phys. A343, 221 (1980)

[5] G Baur, R Rosel, D Trautmann and R Shyam, Phys. Rep. 111, 333 (1984)

[6] Isao Tanihata, J. Phys. G. Nucl. Part. Phys. 22, 157 (1996)

[7] H Machner, Phys. Rep. 127, 309 (1985) 